\title{
Der Analytic Hierarchy Process (AHP): Eine Methode zur Entscheidungsunterstützung im Gesundheitswesen
}

\author{
Axel C. Mühlbacher • Anika Kaczynski
}

Online publiziert: 29. April 2014

(C) The Author(s) 2014. Dieser Artikel ist auf Springerlink.com mit Open Access verfügbar

\begin{abstract}
Problem Regulatory decision bodies and clinical decision-makers have to decide on the approval, pricing and individualized treatment of patients. This decision is based on the assessment of the risk-benefit or cost-benefit ratios. The assessment of benefits often relies on multiple patientrelevant endpoints. Thereby the results of the clinical effects can be contrary. The more endpoints and the more heterogeneous the results, the more complex is the decision-making. The Analytic Hierarchy Process (AHP) is proposed to solve this problem by weighting multiple decision criteria.

Objective The aim of the systematic review was to analyze how the AHP can be used for decision-making in health care in terms of the prioritization of patient-relevant outcomes.

Method Based on a systematic literature review, the study design of the AHP method, applicability and possible limitations for the weighting of patient-relevant outcomes are reported and discussed. The methodological principles are presented and specific concerns during implementation and due to the eigenvalue approach are analyzed.

Results The AHP is suitable to structure group decisions of e.g. different providers, for the management of organizations or the transparent decision-making in regulatory decision bodies. The weakness of AHP is his problematic applicability within large patient populations and the documentation of their preferences. In the future it should be decided to what extent the methodological limitations of the AHP ask for the use of other stated preferences techniques.
\end{abstract}

Zusätzliche Information ist in der online-Version dieses Beitrags (doi:10.1007/s40275-014-0011-8) enthalten.

A.C. Mühlbacher $(\bowtie) \cdot$ A. Kaczynski

IGM Institut Gesundheitsökonomie und Medizinmanagement,

Hochschule Neubrandenburg, Neubrandenburg, Deutschland

e-mail: muehlbacher@hs-nb.de

\section{Multikriterielle Entscheidungsprobleme}

Therapien werden meist ausschließlich aus der Perspektive von klinischen Experten beurteilt. Entscheidungen über die Therapie, auf Grundlage der klinischen Wirksamkeit, werden auf Basis randomisierter klinischer Studien getroffen. Der Fokus liegt auf der klinischen Evidenz. Eine Evidenz hinsichtlich der Patientenpräferenzen ist nicht vorgesehen. Wie Patienten unterschiedliche Eigenschaften einer potentiellen Therapie bewerten, findet kaum Berücksichtigung, weder bei der Zulassung, noch bei der Preisfestsetzung oder leitlinienkonformen Versorgung. Verschiedene Präferenzstudien haben jedoch gezeigt, dass die Urteile der Experten nicht immer mit den Bedürfnissen der Patienten übereinstimmen müssen $[1,2]$. Die Therapieziele der Patienten sind nicht zwangsläufig deckungsgleich mit denen von Experten [3]. Demgegenüber steht die Forderung nach einer zunehmenden Beteiligung der Patienten an medizinischen Entscheidungen, der Stärkung des Mitspracherechts und der Eigenverantwortung [7, 19, 31].

Mit der Forderung nach mehr Selbstverantwortung, Selbstbestimmung und Autonomie vollzieht sich ein $\mathrm{Pa}$ radigmenwechsel. Patienten sollen zukünftig eine aktivere Rolle im Gesundheitssystem einnehmen. Wird das Leistungsangebot an den Präferenzen der Patienten ausgerichtet, kann dies eine Reihe positiver Effekte zur Folge haben. Dies könnte etwa das Verhalten und die Akzeptanz patientengerechter Informations- und Versorgungsstrategien positiv beeinflussen. Sind die wesentlichen Einflussgrößen auf das Entscheidungsverhalten bekannt, kann die Compliance und Adherence gezielt verbessert werden [3]. All dies geschieht in der Hoffnung, die klinischen Erfolgsparameter langfristig zu beeinflussen.

Nicht nur die individuelle klinische Entscheidung sollte sich an den Bedürfnissen des Patienten orientieren, auch 
Krankenkassen und Regulierungsbehörden treffen Entscheidungen, die enorme Auswirkungen auf die individuelle Gesundheitsversorgung und die Bevölkerungsgesundheit haben. Demnach sollten auch diese Entscheidungen die Prioritäten der betroffenen Bevölkerungsgruppen berücksichtigen. Zielsetzung muss es sein, den Patientennutzen zu maximieren. Aus der klinischen Perspektive gilt die evidenzbasierte Medizin (EBM) als Maßstab. Komplexe Entscheidungsprozesse im Gesundheitswesen erfordern dabei die Berücksichtigung mehrerer Zielkriterien. Die Entscheidungsfindung wird umso komplizierter, je mehr Entscheidungskriterien miteinander in Beziehung stehen. Die realisierten Effekte unterschiedlicher Kriterien können sich hinsichtlich der Vorziehenswürdigkeit von Alternativen widersprechen. Infolge der begrenzten menschlichen Informationsverarbeitungskapazität ist der Entscheidungsträger dann häufig nicht in der Lage, unter Berücksichtigung aller relevanten Informationen und Teilaspekte die optimale Lösung zu generieren [13]. Die Gewichtung der unterschiedlichen Zielkriterien bzw. Endpunkte sollte auch aus der Perspektive der Betroffenen erfolgen. Neben der klinischen Evidenz gilt es auch die Prioritäten und Bedürfnisse der Patienten bei der Entscheidungsfindung zu berücksichtigen.

\section{Anwendung: Potentiale des AHP im Gesundheitswesen}

Der AHP ist eine multikriterielle Entscheidungsmethode [14] und wurde in den 1970er Jahren von dem Mathematiker Thomas Saaty entwickelt $[8,15]$. Eingang ins Gesundheitswesen fand der AHP dagegen erst durch Dolan et al. im Jahr 1989 [16, 17]. Diese Methode der Entscheidungsunterstützung wird bis heute überwiegend in den USA und Asien verwendet. In Deutschland konnte sich der AHP noch nicht als Instrument der Entscheidungsfindung etablieren, wird aber seit kurzem im Gesundheitswesen diskutiert $[6,18]$. Es handelt sich beim AHP um einen Prozess, mit dessen Hilfe komplexe und unstrukturierte Entscheidungsprobleme hierarchisch strukturiert werden. So wird der AHP sehr häufig für strategische Managemententscheidungen eingesetzt. Der AHP ist eine Methode der präskriptiven (oder normativen) Entscheidungstheorie, die dem Entscheidungsträger Verfahren und Vorgehensweisen zur Verfügung stellt, um zu einer sinnvollen bzw. nachvollziehbaren (rationalen) Entscheidung zu gelangen [9]. Der Entscheidungsträger löst das Entscheidungsproblem, indem er, basierend auf definierten Zielkriterien und individuellen oder gruppenspezifischen Prioritäten, die nutzenmaximierende Alternative systematisch identifiziert. Ursprünglich wurde die Methode entwi- ckelt, um Entscheidungen kleinerer Gruppen von Entscheidungsträgern zu unterstützen [19].

\subsection{Systematische Recherche}

Im Rahmen dieser Analyse wurde eine systematische Literaturrecherche zur Anwendung des AHP im Gesundheitswesen durchgeführt. Die Recherche in einschlägigen Datenbanken (wie z. B. Pubmed, Springer etc.) wurde anhand des Suchbegriffes ,analytic hierarchy process“ und allen gängigen Abkürzungen in Verbindung mit dem Suchbegriff „health care“ für den Zeitraum 2003-2013 durchgeführt. Insgesamt konnten nach Ausschluss der Studien, in denen die Anwendung des AHPs aus dem Abstract nicht ersichtlich wurde, 87 Referenzen in die Analyse einbezogen werden. Nach der Eliminierung aller nichtenglisch- und nichtdeutschsprachigen Publikationen konnten 71 Quellen identifiziert werden, in denen der AHP zur Untersuchung gesundheitsrelevanter Fragestellungen angewandt wurde. Infolge des Abstract Reviews wurden weitere Referenzen aus der Datenbank entfernt. Es handelte sich dabei um Grundlagenund Übersichtsartikel zur AHP-Methode sowie um Fallstudien, in denen keine empirische Datenerhebung mittels des AHPs durchgeführt wurde. Zur Auswertung standen schließlich 62 Quellen zur Verfügung, aus denen die erstellte Übersicht (siehe dazu das Online-Supplement 1) hervorging. Der Ablauf der systematischen Recherche ist in der Abb. 1 veranschaulicht.

Die Analyse der extrahierten AHP-Studien ergab, dass der AHP in der Mehrheit der Studien zur Unterstützung von Experten- und Managemententscheidungen angewandt wurde $(N=45)$ [32, 55-98]. Der AHP wurde zumeist für Gruppenentscheidungen im Rahmen von Expertenbefragungen verwendet. Die Quellen, welche eine Angabe zur Stichprobengröße beinhalteten, zeichneten sich durch einen geringen Stichprobenumfang aus. Aus dem Literaturreview ging außerdem hervor, dass der AHP bislang eine eher untergeordnete Rolle bei Bürgerbefragungen spielt. So konnten lediglich drei Studien identifiziert werden, in denen eine empirische AHP-Erhebung aus der Bürgerperspektive durchgeführt wurde [99-101]. Im Vergleich zur Anzahl der AHP-Anwendungen für Expertenentscheidungen konnten im Rahmen der Literaturanalyse zudem nur wenige Studien extrahiert werden, die den AHP zur Ermittlung von Patientenpräferenzen nutzten $(N=14)[1,14,19,102-112]$. Es zeigt sich eine klare Tendenz für die Unterstützung von Expertenentscheidungen mittels AHP im Gesundheitswesen [20, 21].

\subsection{Anwendung bei der Entscheidungsfindung}

Im Laufe der Zeit wurden verschiedene Methoden und Modelle der Entscheidungsunterstützung mit dem Ziel einer 
Abb. 1 Ablaufschema der Literaturrecherche

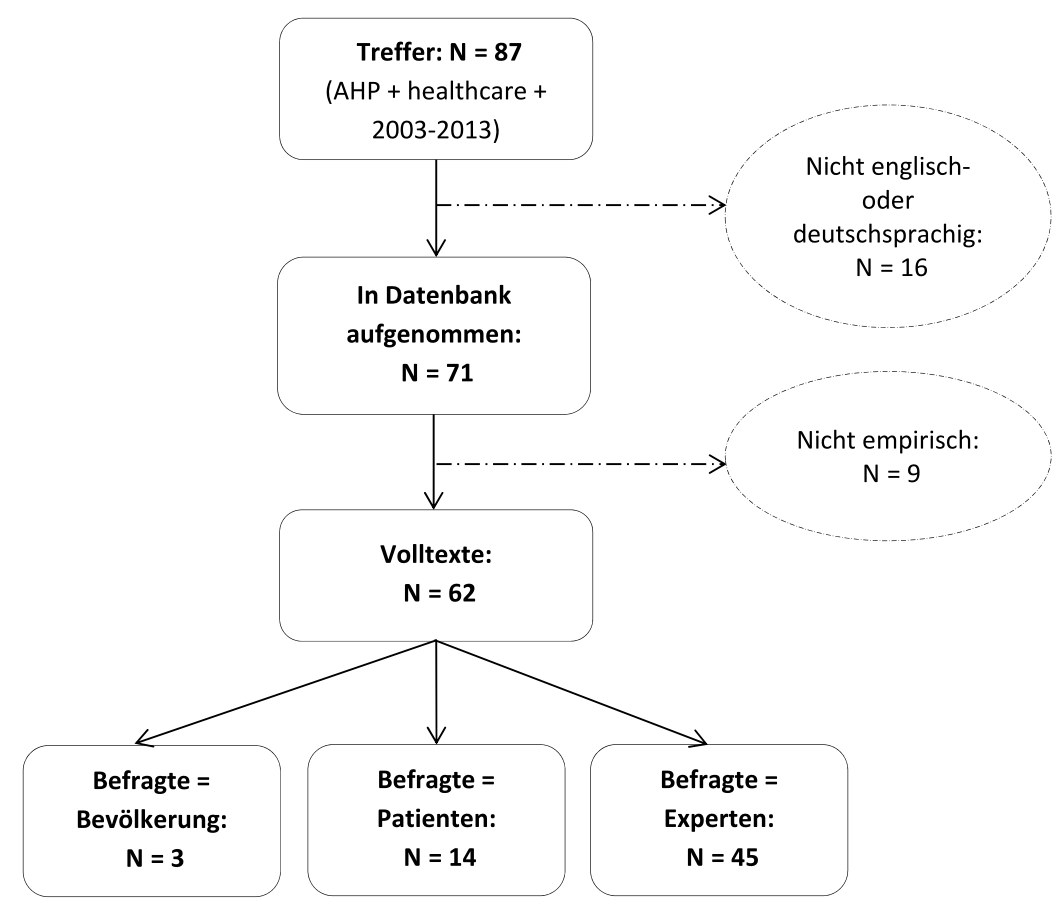

weitestgehend bzw. möglichst rationalen Entscheidungsfindung entwickelt. Die Identifizierung und Gewichtung patientenrelevanter Endpunkte wird bei der Nutzenbewertung medizinisch-klinischer Innovationen seit Längerem gefordert $[4,5]$. Bei der Nutzenbewertung, auf Basis patientenrelevanter Endpunkte, werden derzeit alternative Verfahren der Präferenzmessung durch das IQWiG diskutiert [6]. Neben der klinischen Wirksamkeit soll durch die Analyse der Patientenpräferenzen der patientenseitige Zusatznutzen dokumentiert werden. Durch die Identifizierung und Gewichtung der Entscheidungskriterien kann der klinische Nutzen durch den Patientennutzen ergänzt werden. Erfolgt die Nutzenbewertung auf Grundlage der klinischen Effekte hinsichtlich patientenrelevanter Endpunkte, erhält eine patientenseitige Betrachtung eine enorme Relevanz [7]. Multiple klinische Entscheidungsparameter resultieren in komplexen Entscheidungsproblemen, die durch Methoden der multikriteriellen Entscheidungsanalyse gelöst werden sollen [6]. Zunehmend findet der AHP Anwendung bei medizinischen und gesundheitsökonomischen Fragestellungen zur Lösung multikriterieller Entscheidungsprobleme $[18,20,31]$.

Dieser Beitrag fokussiert sich auf die methodischen Grundlagen, die Studienphasen, das Anwendungspotential sowie die Stärken und Schwächen des Analytic Hierarchy Process (AHP). Die folgenden Ausführungen sind eine Einführung in die Thematik und diskutieren das Potential der Methode im Kontext der Nutzenbewertung von klinischen Entscheidungsträgern oder Regulierungsbehörden.

\section{Methode: Erhebung, Auswertung und Interpretation von AHP-Daten}

\subsection{Studiendesign}

\subsubsection{Definition des Entscheidungsproblems}

Die exakte Definition des Entscheidungsproblems bildet die Basis des AHP [22, 23]. Da der Entscheidungsprozess als Hierarchie dargestellt wird, müssen zunächst alle relevanten Einflussfaktoren sowie Alternativen zur Lösung der Problemsituation erfasst werden. Die Einflussfaktoren, die aus dem Oberziel abgeleitet werden, stellen Unterziele bzw. Kriterien dar. Diese können sich wiederum in weitere Subkriterien gliedern. Schließlich können Alternativen festgelegt werden, die zur Zielerreichung herangezogen werden können [8, 9, 23]. Zur Ermittlung der Kriterien, Subkriterien und Alternativen kann z. B. auf bereits vorhandene (klinische) Studien zurückgegriffen werden. Auch durch umfassende Literaturrecherchen und Rückgriff auf Sekundärmaterial, Expertenbefragungen, Fokusgruppen oder weitere Techniken können Eigenschaften bestimmt werden [12]. Zudem können vor allem im medizinischen Anwendungsgebiet klinische Leitlinien eine Grundlage zur Ermittlung relevanter Einflussfaktoren bieten.

\subsubsection{Hierarchiekonstruktion}

Nach der Identifizierung der Einflussfaktoren erfolgt die Erstellung der Hierarchie, wobei das definierte Oberziel auf 


\begin{tabular}{|c|c|l|}
\hline AHP-Werte & Definition & \multicolumn{1}{c|}{ Interpretation } \\
\hline 1 & Gleiche Bedeutung (Indifferenz) & $\begin{array}{l}\text { Beide miteinander verglichenen Kriterien i und j haben die gleiche Bedeutung } \\
\text { (immer in Bezug auf das Element der nächsthöheren Stufe). }\end{array}$ \\
\hline 3 & Etwas größere Bedeutung & $\begin{array}{l}\text { Erfahrung und Einschätzung sprechen für eine etwas größere Bedeutung des } \\
\text { einen Kriteriums im Vergleich zum anderen Kriterium. }\end{array}$ \\
\hline 5 & Erheblich größere Bedeutung & $\begin{array}{l}\text { Erfahrung und Einschätzung sprechen für eine erheblich größere Bedeutung } \\
\text { des einen Kriteriums im Vergleich zum anderen Kriterium. }\end{array}$ \\
\hline 7 & Sehr viel größere Bedeutung & $\begin{array}{l}\text { Die sehr viel größere Bedeutung des Kriteriums i im Vergleich zum anderen } \\
\text { Kriterium j hat sich in der Vergangenheit klar gezeigt. }\end{array}$ \\
\hline 9 & Absolut dominierend & $\begin{array}{l}\text { Der größtmögliche Bedeutungsunterschied, der } \\
\text { zwischen zwei Kriterien i und j möglich ist. }\end{array}$ \\
\hline $2,4,6,8$ & Zwischenwerte & Feinabstufung \\
\hline $1 / 2,1 / 3,1 / 4,1 / 5,1 / 6,1 / 7,1 / 8,1 / 9$ & $\begin{array}{l}\text { Reziprokwerte für „inverse” Bewertungsurteile, bei denen das Kriterium } j \\
\text { gegenüber dem Kriterium i bevorzugt wird. }\end{array}$ \\
\hline \multicolumn{2}{|r}{} \\
\hline
\end{tabular}

Abb. 2 AHP-Skala (Quelle: Darstellung in Anlehnung an [15])

der obersten Hierarchieebene anzuordnen ist, während die Alternativen auf der untersten Ebene der Hierarchie platziert werden [22, 23]. Die Anzahl der dazwischenliegenden Ebenen der (Sub-)Kriterien ist abhängig vom jeweiligen Entscheidungsproblem und kann nach Bedarf variieren. Hierbei ist zu berücksichtigen, dass jede Ebene der AHPHierarchie von der jeweils darüber liegenden Ebene beeinflusst wird. Demzufolge handelt es sich bei den Subkriterien um Ausprägungen der Kriterien. Die Kriterien stellen wiederum Eigenschaften dar, welche den Entscheidungsgegenstand näher beschreiben. $\mathrm{Zu}$ beachten ist, dass die (Sub-)Kriterien einer Hierarchieebene unabhängig voneinander sein müssen [24].

\subsection{Datenerhebung}

Die Erstellung eines speziellen Erhebungsdesigns ist nicht erforderlich. Beim AHP werden alle Elemente einer Hierarchieebene/-einheit mittels paarweiser Vergleiche zueinander in Beziehung gesetzt. Für die Bildung und Zusammensetzung aller erforderlichen paarweisen Vergleiche siehe Online-Supplement II.

\subsubsection{Bewertung der Hierarchieelemente}

Im Anschluss an die Konstruktion des Erhebungsinstrumentes erfolgt die Bewertung der Elemente (Kriterien, Subkriterien und Alternativen) der Hierarchie. Da die Bewertungen die Grundlage für eine mathematische Lösung des AHP darstellen, müssen diese nummerisch abgebildet werden [22]. Saaty schlägt hierfür die 9-Punkte-Skala vor, die jeweils neun Abstufungen in jede Richtung enthält $[8,9,15,25]$. Die Skalenwerte stellen Verhältniszahlen dar, wobei der Wert 1 den Nullpunkt bildet. Darüber hinaus werden den Skalenwerten verbale Präferenzaussagen zugeordnet (siehe dazu Abb. 2).

Anhand dieser Intervallskala können die AHP-spezifischen paarweisen Vergleiche durchgeführt werden. Dabei werden stets zwei Elemente der Hierarchie einer Ebene in Bezug auf das jeweils übergeordnete Element bewertet [20, 25]. Werden bei der Bewertung umgekehrt gerichtete Präferenzaussagen getroffen, so wird aufgrund der verwendeten Verhältnisskala der reziproke Skalenwert zugeordnet [25]. Aus den paarweisen Vergleichen resultieren dann Bewertungsurteile, welche die Werterelation aus dem Einzelwert des einen Elements und dem Einzelwert eines weiteren Elements bezüglich des übergeordneten Ziels ausdrücken [9].

\subsection{Datenauswertung}

\subsubsection{Berechnung der Gewichte}

In Abhängigkeit von der Struktur der Hierarchie werden sogenannte Bewertungs- oder Vergleichsmatrizen erstellt $[9,25]$. Dazu werden die auf Grundlage der Präferenzurteile ermittelten metrischen Werte in einer Vergleichsmatrix $(A)$ zusammengefasst. Im Anschluss daran werden zur Ermittlung der Prioritäten Matrizenberechnungen durchgeführt. Dabei wird für jedes übergeordnete Hierarchieelement eine Evaluationsmatrix erstellt, welche die Elemente (Kriterien bzw. Subkriterien) der darunterliegenden Ebene als Zeilen- und Spaltenelemente erfasst [24]. So resultiert aus einer Hierarchie mit zwei Ebenen eine Matrix auf erster Ebene, welche die Kriterien in Bezug auf das Oberziel erfasst, sowie „n“ weitere Matrizen für jedes Kriterium, die jeweils alle Ausprägungen des entsprechenden Kriteriums abbilden [22].

In diese Matrizen werden die ermittelten Werte aus den Paarvergleichen übertragen, sodass dabei die Basis- (bzw. Zeilen-) Elemente mit den Vergleichs- (bzw. Spalten-) Elementen in Beziehung gesetzt werden. Die Evaluationsmatrix besteht dabei aus $n \times n$ Elementen, wobei $n$ gleich der Anzahl der Kriterien bzw. Subkriterien ist. Jedes Element der Matrix entspricht einem paarweisen Vergleich und somit einem Präferenzurteil. Die Diagonale der Bewertungsmatrizen stellt den Vergleich der einzelnen Hierarchieelemente 
zu sich selbst dar. Dieser Paarvergleich muss logischerweise nicht ermittelt werden, sondern wird als ,gleichbedeutend“ mit dem Wert 1 belegt [9, 23, 25]. Alle Werte größer als 1 sagen aus, dass das Zeilenelement vorzugswürdiger bzw. wichtiger ist als das Spaltenelement.

Der AHP geht davon aus, dass der Entscheider beim Vergleich eines Merkmals (bzw. einer Merkmalsausprägung) $q$ mit einem Merkmal $p$ zur entgegengesetzten Bewertung kommt als beim Vergleich von Merkmal $p$ gegenüber Merkmal $q$ [10]. Daher wird im Rahmen des AHP auf eine doppelte Befragung verzichtet. Da der AHP annimmt, dass sich die Entscheider „reziprok“ verhalten, kann die Matrixhälfte unterhalb der Diagonalen durch die Bildung der entsprechenden Reziprokwerte vervollständigt werden.

Im Anschluss erfolgt die Berechnung der relativen Gewichte der Kriterien, Subkriterien und Alternativen in Bezug auf das jeweilige Element der darüber liegenden Hierarchieebene. Die relativen Wichtigkeiten oder Zielgewichte, die aus den Paarvergleichsmatrizen abgeleitet werden, können dabei mit Hilfe der Eigenvektorberechnung ermittelt werden [8, 23, 34]. Neben der meistens angewendeten Eigenwertmethode existieren auch eine Reihe weiterer Methoden, die zur Schätzung der Prioritäten angewandt werden können. Dazu zählt u. a. das „Goal Programming“, die „Logarithmische Least Squares Methode" (LLSM) oder auch das „Least Squares Verfahren“ (LSM) [9, 26]. Für eine alternative Möglichkeit zur Bestimmung der Zielgewichte sei an dieser Stelle auf Chandran/Golden/Wasil (2005) verwiesen [27].

\subsubsection{Ermittlung der Gewichtungsvektoren}

Zur Bestimmung der Gewichte werden im ersten Schritt die Spaltensummen $\left(c_{i}\right)$ der Bewertungsmatrizen gebildet. Anschließend werden die Paarvergleichswerte durch die ermittelte Spaltensumme dividiert, sodass eine Normierung der Evaluationsmatrix auf 1 erfolgt. Aus dieser Berechnung resultiert die normalisierte Matrix, deren Zeilenelemente zu Zeilensummen $\left(r_{i}\right)$ aufaddiert werden. Zur Ermittlung der sogenannten lokalen Kriterien- bzw. alternativen Gewichte werden die Zeilensummen durch die Anzahl der Elemente (Kriterien oder Subkriterien) dividiert. Dieser ermittelte Wert, der dem rechten Haupt-Eigenvektor (principal right eigenvector) entspricht, bildet dann das Gewicht $(w)$ für das entsprechende Element.

Die Berechnung der Prioritäten stellt einen iterativen Prozess dar. Die Eigenwertmethode erfolgt hierbei mittels der Potenzmethode in einem dreistufigen Verfahren. Dazu wird die Evaluationsmatrix in einem ersten Schritt quadriert, d. h. die Matrix wird entsprechend der mathematischen Matrizenrechnung mit sich selbst multipliziert. Dafür wird die Zeile $i$ der Matrix mit der Spalte $j$ der gleichen Matrix paarweise multipliziert und die Produkte zu dem jeweiligen
Element $i, j$ der Produktmatrix aufsummiert [16]. AnschlieBend wird die Matrix normalisiert, indem die Werte durch die Spaltensummen dividiert werden. Dann werden die lokalen Gewichte berechnet.

Dieser Prozess der Quadrierung, Normalisierung und Gewichtsberechnung wird solange wiederholt, bis sich die berechneten relativen Gewichte nur noch geringfügig bzw. in der Höhe des zuvor ermittelten Wertes der zuvor potenzierten Matrix unterscheiden [8, 23, 28]. Dabei verringern sich die Abweichungen zwischen den berechneten Gewichten mit zunehmender Potenz, sodass fortschreitend eine Annäherung an die tatsächlichen relativen Gewichte erfolgt. Demzufolge endet die Berechnung der sog. lokalen Gewichte, wenn die Differenz zwischen den Werten nur noch sehr gering ausfällt.

\subsubsection{Konsistenzprüfung}

Eine Entscheidung wird als konsistent bzw. transitiv bezeichnet, wenn z. B. A zweimal wichtiger als B, B dagegen dreimal wichtiger als $C$ und $A$ folglich sechsmal wichtiger als C ist [29]. Die Annahme der Transitivität erfordert die Widerspruchsfreiheit der Präferenzordnung. Somit ist die Bewertungsmatrix konsistent, wenn $a_{i j} \times a_{j k}=a_{i k}$ für beliebige $i, j$ und $k$ gilt [13]. Aufgrund der beim AHP geforderten Präzisierung der Bewertungsurteile und der begrenzten Informationsverarbeitungskapazität ist ein Entscheider jedoch häufig nicht fähig, vollständig konsistente Werturteile $\mathrm{zu}$ treffen. Allerdings sind geringfügige Abweichungen von der Konsistenz beim AHP bis zu einem gewissen Grad unbedenklich und können akzeptiert werden [24]. Die Konsistenzprüfung ist ein AHP-spezifischer Verfahrensschritt, der im Anschluss an die Bewertung auf Basis der Evaluationsmatrix durchgeführt wird. Die Stärke der Inkonsistenz gibt dabei Aufschluss über die Validität der Ergebnisse [30].

Beim AHP kann die Konsistenz der Evaluationsmatrix mit Hilfe des maximalen Eigenwertes (principal eigenvalue) gemessen werden [8]. Der maximale Eigenwert $\lambda_{\max }$ entspricht bei einer vollständig konsistenten Entscheidung immer der Summe der Spaltenelemente $n$ des gesuchten Eigenvektors. Der Konsistenzindex $C I$ (consistency index) wird wie folgt ermittelt.

Es gilt: $\quad C I=\frac{\lambda_{\max }-n}{n-1}$

Durch den Vergleich zwischen $C I$ und einem Durchschnittswert $R$ (für „Random“), der Erfahrungswerte darstellt, die empirisch in Testreihen nachgewiesen wurden, kann festgestellt werden, inwieweit die Inkonsistenz (bzw. die Abweichung von der Konsistenz) noch akzeptiert werden kann. Dabei ist die Konsistenz der Bewertungen auch abhängig von der Größe der Evaluationsmatrix. So steigt die Wahrscheinlichkeit für inkonsistente Antworten mit zunehmender Anzahl an Elementen bzw. Paarvergleichen. Somit erhöht sich der durchschnittliche Konsistenzindex $R$, je größer 
Tab. 1 Durchschnittlicher Konsistenzindex $R$ bei gegebener Matrixgröße [8]

\begin{tabular}{lllllllllll}
\hline Größe der Matrix & 1 & 2 & 3 & 4 & 5 & 6 & 7 & 8 & 9 \\
Konsistenzindex $R$ & 0,00 & 0,00 & 0,52 & 0,89 & 1,11 & 1,25 & 1,35 & 1,40 & 1,45 & 1,49 \\
\hline
\end{tabular}

$n$ ist. Tabelle 1 zeigt den Konsistenzindex $R$ in Abhängigkeit von der Matrixgröße.

Mittels der Konsistenzverhältniszahl $C R$, die aus dem Verhältnis zwischen $C I$ und $R$ hervorgeht $\left(C R=\frac{C I}{R}\right)$, kann die Größe der Evaluationsmatrix entsprechend Berücksichtigung finden [8]. Nach Saaty ist eine Evaluationsmatrix ausreichend konsistent, wenn $C R<0,1$ ist [8]. Andere Autoren sind der Auffassung, dass bei komplexen Hierarchien und solchen, die einer unmittelbaren Modifikation während des Bewertungsvorganges nicht zugänglich sind, auch ein Grenzwert von 0,2 akzeptiert werden kann [14, 31, 32]. Überschreiten die Konsistenzmaße den gegebenen Richtwert, sollten die Hierarchieelemente erneut bewertet bzw. die Hierarchie vollständig überarbeitet werden.

\subsection{Dateninterpretation}

\subsubsection{Ermittlung der Zielgewichte und Festlegung der Rangfolge}

Anschließend werden die einzelnen Aussagen zu einem Gesamturteil hinsichtlich der Relevanz der Lösungsalternativen für die Zielerreichung operationalisiert. Die Teilgewichte werden zu einem Gesamtgewicht für jede Alternative zusammengeführt $[9,25]$. Dabei werden über die gesamte Zielhierarchie die Gewichtungsvektoren der Alternativen für jedes Ziel bestimmt. Die AHP-Hierarchie wird daher schrittweise mit Hilfe eines Bottom-up-Vorgehens interpretiert [25]. Die Ermittlung der Teilgewichte erfordert die Bestimmung globaler Zielgewichte. Dafür werden aus den bereits ermittelten lokalen Gewichten (der einzelnen Hierarchieelemente in jeder Merkmalsebene) die globalen Gewichte aggregiert [25, 33]. Das globale Gewicht eines Hierarchieelementes zeigt die korrekte Bedeutung des jeweiligen Hierarchieelementes im Kontext der Gesamthierarchie [25]. So ist unter einem globalen Bedeutungsgewicht die relative Wichtigkeit eines Elements im Vergleich zu allen anderen Elementen zu verstehen. Ein lokales Gewicht drückt lediglich die relative Bedeutung eines Elements im Vergleich zu den Elementen der gleichen Rubrik aus [25]. Zur Bestimmung der globalen Gewichtungsvektoren wird das lokale Zielgewicht der Kriterien/Alternativen einer unteren Ebene jeweils mit dem lokalen Zielgewicht des Kriteriums der darüber liegenden Ebene multipliziert.

Anschließend erfolgt die Berechnung der globalen Gewichte der Alternativen. Dazu werden die lokalen Gewichte der Alternativen mit den globalen Gewichten der dar- über liegenden (Sub-)Kriterien multipliziert [22]. Dann erfolgt die Berechnung der Gesamtgewichte der Alternativen, indem die zuvor ermittelten Teilgewichte der Alternativen aufaddiert werden. Die Summe der so berechneten globalen Gewichte der Alternativen ergeben den Prioritätsindex, der das Gesamtgewicht jeder Alternative darstellt [22]. Über die Höhe des Gesamtwertes lässt sich in der Folge die beste Alternative ermitteln. Dieses errechnete Gewicht ist somit die (vorläufige) Lösung des AHP und begründet die Rangreihenfolge der Alternativen. Aus diesem Wert geht hervor, mit welchem Gewicht (bzw. wie viel) die einzelnen Lösungsalternativen zur Zielerreichung beitragen. Somit kann aus der Errechnung der Gesamtgewichte je Alternative interpretiert werden, wie wichtig bzw. vorzugswürdig die einzelnen Alternativen sind. Folglich wird diejenige Alternative präferiert, die den höchsten Gesamtwert aufweist. Diese Alternative erhält somit den ersten Rang. Die Alternative mit dem zweithöchsten Wert erhält den zweiten Rang etc., bis jede Alternative einem Rang zugeordnet wurde [9].

\subsubsection{Sensitivitätsanalyse}

Mittels der Sensitivitätsanalyse soll untersucht werden, in welchem Maße Änderungen in der Gewichtung der Kriterien (bei gleichbleibender Modellstruktur) Auswirkungen auf die Reihenfolge der Lösungsalternativen haben. Durch die kontinuierliche Veränderung der Kriteriengewichtungen werden sensitive Grenzen definiert, bei denen sich die Rangreihung der Alternativen umkehrt. Das bedeutet, dass anstelle der aktuell zu wählenden Lösungsalternative eine andere Alternative ausgewählt werden würde. Ein stabiles Ergebnis liegt vor, wenn marginale Änderungen der Gewichte das Endergebnis nicht beeinflussen. Ist der Abstand zwischen den aktuellen Merkmalsgewichten und den Grenzen gering, so deutet dies auf ein instabiles Ergebnis hin, was eine Prüfung oder die Wiederholung des Beurteilungsprozesses bzw. des gesamten AHP erfordern kann. Konnten stabile Ergebnisse bestätigt werden, gibt die abschlieBende Bewertung der Alternativen die endgültige Präferenzordnung der Lösungsalternativen an [8, 33, 46].

\subsubsection{Gruppenentscheidung}

Für die Berücksichtigung der Bewertungen mehrerer Personen ist es erforderlich, die Gewichte zu einer Gruppenentscheidung zu aggregieren [22]. Zur Verdichtung der Einzelentscheidung bieten sich grundsätzlich verschiedene Algorithmen wie z. B. der arithmetische und der geometrische 
Mittelwert, der Modalwert oder aber auch der Median an. Für den AHP wird der geometrische Mittelwert empfohlen, da dieser die Bedingung erfüllt, dass die aggregierten Werte der oberen diagonalen Matrixhälfte den reziproken Werten der unteren diagonalen Matrixhälfte entsprechen [33]. Demzufolge wäre das geometrische Mittel auch der einzig zulässige Algorithmus, der im Rahmen des AHP zur Aggregation eines Gruppenurteils angewandt werden könnte. Dieser Algorithmus kann folglich herangezogen werden, um individuelle Urteile mehrerer Entscheidungsträger als Ganzes in den Entscheidungsprozess zu integrieren [14, 19].

\section{Diskussion: Vorteile und Limitationen des AHP}

Aufgrund der hohen Flexibilität zeichnet sich diese Methode durch einen sehr breiten Anwendungskontext aus. Die traditionelle Anwendung des AHP zur strukturierten Analyse von Management- und Gremienentscheidungen wurde auf vielfältige Einsatzgebiete übertragen. Nach Saaty [36] weist der AHP eine Vielzahl von Stärken auf, welche hier kurz zusammengefasst werden.

Der AHP ist ein leicht verständliches, flexibles Modell und vereint die deduktive Vorgehensweise mit dem Systemansatz zur Lösung komplexer und unstrukturierter Probleme. Durch die hierarchische Struktur der Ziele kann der AHP mit der Interdependenz von Systemelementen unterschiedlicher Zielhierarchien umgehen und besteht nicht aus linearen Denkmustern. Der AHP berücksichtigt die natürliche kognitive Tendenz, Elemente in Kategorien zu ordnen und ähnliche Elemente auf jeder Hierarchieebene zu gruppieren. Durch die Bewertungsskala ist es außerdem möglich, sowohl quantitative als auch qualitative Merkmale innerhalb eines Entscheidungsproblems zu berücksichtigen. Damit liefert der AHP eine Methode zur umfassenden Ermittlung von Prioritäten auf Basis einer Skala zur Messung immaterieller Dinge. Darüber hinaus besteht die Möglichkeit nicht nur individuelle Einzelurteile in den Entscheidungsprozess zu integrieren, sondern auch für Gruppenentscheidungen eine Ergebnisverdichtung zu generieren [21]. Beim AHP besteht keine Notwendigkeit stets einen Konsens zu erreichen, sondern er verdichtet ein repräsentatives Ergebnis aus einer Reihe von Beurteilungen. Ein weiterer Vorteil des AHP ist die Integration des Konsistenztestes und der Sensitivitätsanalyse mit deren Hilfe die Qualität der Ergebnisse, insbesondere die Widerspruchsfreiheit der Bewertungen sowie die Stabilität der Ergebnisse, überprüft werden kann. Damit berücksichtigt der AHP die logische Konsistenz von Beurteilungen, die zur Ermittlung der Prioritäten verwendet werden. Ein weiterer Vorteil wird darin gesehen, dass der AHP es dem Anwender ermöglicht, die Problemdefinition zu verfeinern und die Beurteilungen und das Problemverständnis durch Wiederholung zu verbessern. Die Konsistenz der Befragung wird nicht selten durch eine Wiederholung der Fragen verbessert. Eine weitere Stärke des AHP ist, dass er eine qualitative Verbesserung der Entscheidungsfindung ermöglichen kann [35]. Der AHP führt zu einer generellen Schätzung der Vorzugswürdigkeit einer jeden Alternative, da er die relative Wichtigkeit von Faktoren eines Systems berücksichtigt und damit ermöglicht, dass die besten Alternativen auf Basis der Ziele und deren Bewertungen durch die Entscheider erfolgt.

Im Zuge der Übertragung auf neue Forschungsfragen stieg auch die Kritik. Über die Jahre wurden dem AHP auch eine Reihe von theoretischen und methodischen Schwächen zugeschrieben, sodass das Studiendesign, die Methode und die Durchführung bis heute an wesentlichen Punkten kritisiert werden [13, 37-40].

\subsection{Studiendesign}

\subsubsection{Fehlende theoretische Fundierung und Akzeptanz von Inkonsistenzen}

Ein Nachteil der Methodik ist, dass irrationale Entscheidungen häufig nicht als falsche Entscheidungen identifiziert werden [46]. Der Grund dafür ist, dass der AHP auf die restriktiven und nutzentheoretisch fundierten Annahmen hinsichtlich der Konsistenz und Transitivität verzichtet [40]. Der AHP weist somit keine nutzentheoretische Fundierung auf. Grundlegende Anforderungen der ökonomischen Nutzentheorie werden hierbei verletzt. Somit steht auch die Annahme der Axiome, die das theoretische Fundament des AHP bilden, häufig im Zentrum der Kritik [38]. Ferner werden den Verbindungen der Punktwerte sowie den entsprechenden verbalen Umschreibungen in der Literatur eine fehlende theoretische Fundierung unterstellt [39].

\subsubsection{Offene Interpretation der Fragestellung}

Die AHP-spezifische Fragestellung bei der Nutzenbewertung kann lauten: „Welche Eigenschaft hat eine höhere Bedeutung/Wichtigkeit für Ihre Therapieentscheidung?" Bei traditionellen AHP-Anwendungen wird oft bei allen Entscheidungskriterien ein positiver Nutzen unterstellt. Im Kontext der klinischen Nutzenbewertung werden meistens im Entscheidungsmodell die positiven Effekte (z. B. Heilung) den negativen Auswirkungen der Therapie (z. B. Nebenwirkungen) gegenübergestellt. Dies kann Probleme bei der Interpretation der Fragestellung mit sich bringen. Es kann unklar bleiben, ob eine Eigenschaft bevorzugt wird, da der Entscheider einen hohen Nutzengewinn erwartet oder sich die Relevanz aus dem zu erwartenden hohen Nutzenverlust ableitet. Die sehr abstrakte Frage nach der Wichtigkeit im Paarvergleich bietet einen (zu) großen Interpretationsspielraum. 
Primär ist beim AHP nicht vorgesehen, dass auf der Ebene der Eigenschaften (hier der Beschreibung der patientenrelevanten Endpunkte) die Ausprägung dokumentiert wird. Dies erfolgt auf der nächsten Bewertungsstufe. Ohne eine konkrete Beschreibung der Endpunkte ist aber eine Beurteilung der Relevanz nicht möglich. Ohne eine exakte Benennung der Ausprägungsgrade werden die Entscheider subjektiv eine Annahme treffen. Eine Aggregation der Daten ist fraglich, da davon ausgegangen werden kann, dass jeder Entscheider eine unterschiedliche Ausprägung angenommen hat. Diese Annahmen über den Ausprägungsgrad dürften unter anderem von den bisherigen Erfahrungen abhängig sein und sind damit Ursache für eine zunehmende, nicht erklärbare Heterogenität im Antwortverhalten. Angenommen, es wird eine Eigenschaft durch einen festen Ausprägungsgrad bei der Befragung fixiert. Selbst in diesem Fall ist weiterhin von einem großen Interpretationsspielraum auszugehen. Beim direkten Paarvergleich von zwei Nebenwirkungen könnte diejenige für den Teilnehmer von Bedeutung sein, welche niedrig ausfällt und deshalb in Kauf zu nehmen ist. Aus dieser Perspektive fällt die Entscheidung auf Kriterien, welche das geringere „Übel“ erwarten lassen. Die Fragestellung des AHP kann auch entgegengesetzt interpretiert werden: Es ist denkbar, dass die jeweils am bedrohlichsten empfundene Eigenschaft einer Therapie angezeigt wird, da im Hinblick auf eine mögliche Therapieentscheidung dieser Ausprägung eine größere bzw. höhere Bedeutung zugemessen wird. Diese Art der Interpretation ist ebenfalls nachvollziehbar. Ein hohes Risiko einer Nebenwirkung führt dazu, dass eine andere Therapiealternative gewählt wird.

Infolge dieser verschiedenen Interpretationsweisen besteht die Gefahr, dass die Probanden (in diesem Kontext zumeist Patienten) ihr Präferenzurteil entgegen der eigentlichen Intention bzw. Zielsetzung des AHPs fällen. Dieses Wahlverhalten kann innerhalb eines Interviews korrigiert werden, wobei bei Erhebungen über das Internet oder Fragebögen eine Korrektur nur sehr schwer möglich ist.

\subsection{Datenerhebung}

\subsubsection{Bewertung von Paarvergleichen}

Die Bewertung von Paarvergleichen entspricht keiner realen Entscheidungssituation, da stets nur zwei Einzelaspekte einer Entscheidung gegeneinander abgewogen werden. Somit weist das Frageformat des AHP zumeist nur eine geringe Übereinstimmung mit realen Entscheidungssituationen von Patienten und Ärzten auf. Da die paarweisen Vergleiche jeweils nur innerhalb einer Hierarchieebene erfolgen, wird dem Entscheider zu keinem Zeitpunkt die Gesamtsituation ersichtlich. Reale Entscheidungssituationen, z. B. die Bewertung von Arzneimitteln hinsichtlich der Effekte, Nebenwirkungen oder Art der Anwendung, können somit nicht adäquat abgebildet werden [18]. Auch wenn der aktuell erschienene IQWiG-Bericht zum AHP-Pilotprojekt zu dem Ergebnis kommt, dass die Datenerhebung der AHP für Patienten handhabbar ist [52], ist fraglich, inwieweit eine Verständlichkeit des Frageformats aufgrund der realitätsfernen und abstrakten Entscheidungen vorausgesetzt werden kann [25]. Unabhängig davon könnte die abstrakte Erhebung von Experten genutzt werden. Dies bestätigt sich auch bei der beschriebenen Literaturrecherche.

\subsubsection{Bewertungskompetenz der Probanden}

Die AHP sieht vor, dass mittels der 9-er Skala differenzierte Auswahlentscheidungen durch den Probanden (z. B. Patienten) getroffen werden. Im direkten Paarvergleich soll der Befragte eine Auswahlentscheidung treffen, die im Ergebnis aussagt, wie sehr die befragte Person ein Zielkriterium gegenüber einem anderen bevorzugt. Der Teilnehmer der Befragung muss angeben können, um wie viel wichtiger ein patientenrelevanter Endpunkt im Vergleich zum jeweils anderen ist. Diese Aussagen mögen für einen Experten eventuell noch möglich, einfach und plausibel sein, wenn beispielsweise Eigenschaften eines alltäglichen Konsumgutes oder die Merkmale einer gewünschten oder erfahrenen (Dienst-)Leistung beurteilt werden sollen. Wird allerdings von einem Patienten oder Versicherten erwartet, Werturteile hinsichtlich gesundheits- bzw. krankheitsbezogener Güter oder Leistungen zu treffen, so können erhebliche Schwierigkeiten bei der derart differenzierten Bewertung auftreten. Probanden könnten nicht oder zumindest nur sehr begrenzt in der Lage sein, Aussagen dahingehend zu treffen, inwiefern ein bestimmtes Merkmal zweimal, dreimal oder viermal etc. so wichtig ist wie ein anderes Merkmal. So ist in der Realität ein Patient kaum fähig zu sagen, dass er z. B. Lebensverlängerung neun Mal mehr präferiert als Kopfschmerzen. Die meisten Patienten sind somit nicht in der Lage die relative Bedeutung, die sie einem Endpunkt beimessen, detailliert bzw. exakt in seiner Intensität zu bestimmen. Dieser Limitation begegnet der AHP durch Erweiterung der nummerischen Skala um die verbalen Präferenzaussagen (siehe dazu auch Abb. 2). Durch die Kombination aus qualitativen und quantitativen Skalenwerten wird den Probanden die Bestimmung der relativen Bedeutung eines Merkmals zum Teil erleichtert. Der AHP verlangt somit nicht zwangsläufig eine exakte nummerische Aussage zur relativen Wichtigkeit. Dennoch liegt die Vermutung nahe, dass kranke Menschen mit dem Rating von Leveln, Attributen und Alternativen kognitiv überfordert sind. Folglich kann es während der Bewertung (Rating) im AHP aufgrund der sehr anspruchsvollen Bewertungsaufgabe zu Heuristiken und Artefakten kommen, da mit begrenztem Wissen, Mutmaßungen und wenig Zeit eine Entscheidung gefällt wird [12, 54]. 


\subsubsection{Begrenztheit der AHP-Skala}

Die 9-Punkte-Bewertungsskala kann nicht alle Bewertungen, die durch einen Befragungsteilnehmer geäußert werden können, wiedergeben [9, 13]. Infolge der Begrenztheit der Bewertungen können inkonsistente Bewertungen systematisch generiert werden [37]. Wird z. B. Merkmal A gegenüber Merkmal B mit drei Punkten bewertet und Merkmal B gegenüber Merkmal C mit fünf Punkten, so ist im Rahmen der Skala keine konsistente Bewertung von Merkmal A gegenüber Merkmal C möglich, da der Wert 15 nicht auf der Skala enthalten ist. Somit resultieren aus der Skala inkonsistente Werturteile [25, 39]. Verschiedene Untersuchungen auf diesem Gebiet aber zeigen auch, dass Probanden zumeist mit der Vielzahl an feinen Abstufungen überfordert sind [25]. Hinzu kommt, dass die Befragten häufig nur die extremen Skalenpunkte (hier: 9 oder 1) wählen. Eine Möglichkeit zur Vermeidung dieser Problematik besteht darin, die Skala zu modifizieren [25]. So könnten die Zwischenwerte, die der Abstufung eines Präferenzurteils dienen, eliminiert werden, sodass die Skala insgesamt nur neun Ausprägungspunkte umfasst [25].

\subsection{Datenauswertung}

\subsubsection{Inkonsistenz der Präferenzurteile}

Wie verschiedene Studien zeigten, sind viele Patienten nicht in der Lage, durchgängig widerspruchsfreie Bewertungen vorzunehmen. Die von Saaty vorgeschlagene Inkonsistenzgrenze von 0,1 kann daher nur selten bei Befragungen unterschritten bzw. erreicht werden. Wie bereits dargelegt, müssen zur Ermittlung der aggregierten Gewichte üblicherweise alle Einzelbewertungen, die einen Konsistenzindex von $C R=0,1$ überschreiten, aus der Auswertung ausgeschlossen werden. Allerdings kann die Konsistenz der Bewertungen im Rahmen schriftlicher Befragungen nicht unmittelbar (bzw. zum Zeitpunkt der Datenerhebung) überprüft werden. Daher ist es nicht möglich, im Falle nicht hinreichender Konsistenz die Paarvergleiche zu modifizieren und die Bewertung zu wiederholen, so wie es von Saaty ursprünglich vorgeschlagen wurde. Daher ist eine zulässige Inkonsistenzgrenze von maximal $10 \%$ in schriftlichen Befragungen eher unangemessen. In Anlehnung an vergleichbare Studien wird daher oft ein $C R \leq 0,2$ zu Grunde gelegt und akzeptiert [14, 31, 32]. Allerdings besteht auch die Möglichkeit die Präferenzdaten der Probanden in Fokusgruppen bzw. in Gruppendiskussionen zu ermitteln. Diese Vorgehensweise, wie sie auch im IQWiG-Pilotprojekt zur AHP-Methode verwendet wurde, bietet den Vorteil, dass die Probanden im Anschluss an einen bewerteten paarweisen Vergleich, ihr Urteil, infolge der nach jeder Bewertungsrunde stattfindenden Gruppendiskussion, noch einmal verändern können [52]. So kann im Falle einer inkonsistenten Bewertung das Urteil überprüft oder revidiert werden. Allerdings gibt es in Gruppendiskussionen zumeist eine bzw. einige wenige dominante Personen, welche die Meinungen und Ansichten der anderen Mitglieder sehr stark beeinflussen und sich mit ihren Einschätzungen durchsetzen. Folglich können die Ergebnisse nie das Meinungsspektrum aller Befragungsteilnehmer widerspiegeln. Außerdem kann sozial erwünschtes Antwortverhalten hervorgerufen werden, wodurch Verzerrungen entstehen, die kaum kontrolliert werden können [54]. $\mathrm{Zu}$ diskutieren bleibt, inwiefern diese Einflussnahme der Interviewer im Prozess des AHP die Ergebnisse beeinflusst, wie diese Einflussnahme transparent gestaltet und ob letztendlich der Ausschluss oder die Beeinflussung toleriert werden kann.

\subsubsection{Rank Reversal}

Eine weitere methodische Schwäche ist die Umkehr der Rangreihung. Kritisiert wird, dass es durch das Hinzufügen bzw. Entfernen weiterer (irrelevanter) Alternativen, zu einer Änderung der Rangfolge der Lösungsalternativen kommen kann. Stehen beispielsweise die zwei Alternativen A und B zur Auswahl, wäre es möglich, dass Alternative A der Alternative $\mathrm{B}$ vorgezogen wird. Wird eine weitere Alternative (Alternative $\mathrm{C}$ ) als Lösung hinzugefügt, kann sich die Rangfolge ändern, sodass die Lösungsalternative B der Alternative A vorgezogen wird [13]. Demzufolge ändert sich die Rangordnung der Alternativen, obwohl die Urteile der Paarvergleiche bezüglich der ursprünglichen alternativen Menge beibehalten werden [41]. Die Bewertung der optimalen Lösungsalternative kann durch die Ausprägung einer neuen Alternative verringert werden, ohne dabei selbst eine optimale Lösungsalternative zu werden oder gewesen zu sein [42]. Kritiker zweifeln folglich an der Stabilität der Resultate und unterstellen, dass die Ergebnisse einer gewissen Willkür geschuldet seien [13, 37]. Die Annahme der Rationalität wird nach Auffassung verschiedener Autoren verletzt, da die Ergebnisse unter bestimmten Voraussetzungen von irrelevanten Lösungsalternativen abhängig sind. Zudem können infolge der Änderung der Rangfolge, die nicht auf geänderten Bewertungen der Befragten, sondern einzig und allein auf die Auswertungsmethodik zurückzuführen sind, Fehlurteile generiert werden [13, 37, 42].

Das Rangumkehrproblem beim AHP wird vor allem dadurch verursacht, dass die Zusammenhänge zwischen der Skalierung der lokalen Prioritäten auf einer Stufe und der Erhebung der Urteile auf einer nächsthöheren Stufe nur unzureichend berücksichtigt werden [41, 43, 44]. Dies fällt gerade bei der Gewichtung von Endpunkten ins Gewicht. Die Eigenvektormethode des AHP, insbesondere die Summennormierung, ist die Ursache der Rangumkehr [37]. So ändert 
sich durch das Hinzufügen einer weiteren Alternative die absolute Differenz der Ausprägungen, da die Normierungsprozedur zu einer veränderten Verteilung der lokalen Prioritätswerte aller anderen Alternativen führt. Um Rank Reversal beim AHP zu vermeiden, wurden in der Literatur zahlreiche Ansätze und Erweiterungen vorgeschlagen und diskutiert, wie z. B. der „B-G modified AHP“, das ,Supermatrixverfahren“, der sog. „Referenced AHP“ [45] oder der „Normalization to minimum entry"-Ansatz. Zudem stellt die Sensitivitätsanalyse eine Möglichkeit dar, die Rangumkehr durch die Änderung der Gewichte zu simulieren. Zwar kann Rangreversion nicht ausgeschlossen werden, dennoch ist die Sensitivitätsanalyse von grundlegender Bedeutung für die effektive Verwendung und Implementierung quantitativer Entscheidungsmodelle.

\subsection{Dateninterpretation}

\subsubsection{Die Unabhängigkeit der Attribute}

Dem AHP wird als Wertmodell häufig die gewichtete additive Wertfunktion zu Grunde gelegt [11, 18, 53]. Folgt man dieser Annahme, so kann der AHP nur dann zuverlässig angewandt werden, wenn voneinander unabhängige Eigenschaften verwendet werden. Potentielle Interaktionseffekte zwischen den zu bewertenden Eigenschaften müssen somit ausgeschlossen werden [30]. Wird der AHP zur Messung der Prioritäten von Patienten oder Versicherten angewandt, handelt es sich bei den Eigenschaften (Kriterien) meist um patientenrelevante Endpunkte wie z. B. Mortalität (z. B. Sterberisiko), Morbidität (z. B. Herzinfarktwahrscheinlichkeit) und Nebenwirkungen (z. B. Kopfschmerzen) oder Lebensqualitätsparameter (z. B. Aktivitäten des täglichen Lebens). Die Endpunkte Morbidität und Mortalität sind in der Therapie unmittelbar relevant für den Patienten. Dennoch können diese Eigenschaften im Einzelfall nur begrenzt kausal interpretiert werden. Sowohl für den Bereich der Krankheitsursachen als auch in Hinblick auf Behandlungsergebnisse und Eigenschaften von Therapien ergeben sich eine Reihe von Abhängigkeiten, die bei der Formulierung des Entscheidungsmodells beachtet werden müssen. Auch im aktuellen AHP-Pilotprojekt des IQWiG werden Überlappungen bis zu einem bestimmten Grad als unvermeidbar angesehen [52]. Die Autoren weisen darauf hin, dass Korrelationen und Überlappungen zwischen den Endpunkten (bzw. Therapiezielen) zu einer Über- und Unterschätzung der Kriteriengewichte führen können. Außerdem kann durch die Verwendung abhängiger Attribute die Bewertung der Paarvergleiche äußerst schwierig oder gar unmöglich werden, was eine Verzerrung der Ergebnisse zur Folge haben kann [52].

Stellt man allerdings das additive Wertemodell in Frage und legt dem AHP ein anderes Wertemodell zugrunde, so kann ein gewisser Grad an Abhängigkeit der Eigenschaften legitimiert werden. Somit könnte dieser Ansatz das Nichtbestehen einer vollständigen Unabhängigkeit zwischen den Attributen zum Teil rechtfertigen. Auch im Arbeitspapier zum AHP-Pilotprojekt wird argumentiert, dass ein gewisses $\mathrm{Ma} ß$ an Abhängigkeit im AHP-Verfahren akzeptiert werden kann [52]. Die Forderung unabhängiger Attribute wird vor allem bei klinischen Fragestellungen, z. B. bei der Gewichtung patientenrelevanter Endpunkte, nur sehr schwer zu realisieren sein. Aufgrund dieser anwendungsspezifischen Schwierigkeiten muss die Sachdienlichkeit des AHP für die indikationsspezifische Präferenzmessung im Kontext der Nutzenbewertung in Frage gestellt bzw. kritisiert werden. Um die Problematik, die durch die Verwendung abhängiger Eigenschaften hervorgerufen wird, zu umgehen, besteht jedoch Möglichkeit, anstelle des AHP das sog. ANP-Verfahren anzuwenden. Dabei handelt es sich um eine erweiterte Form der AHP-Methode, welche die Berücksichtigung bestehender Abhängigkeiten zwischen den Kriterien (bzw. Endpunkten) erlaubt [10, 52].

\subsubsection{Grenzwertbetrachtung}

Der AHP lässt keine Grenzwertbetrachtung zu. Rückschlüsse hinsichtlich der relativen Wichtigkeit von Attributen im Sinne der ökonomischen Analyse (Grenzrate der Substitution) sind nicht möglich. Der Grund dafür ist, dass der Grenznutzen eines Attributs jeweils vom Wert des anderen Attributs (allgemeiner: der anderen Attribute) abhängt und somit Interaktionen zwischen den Attributen existieren. Dieser Zusammenhang lässt sich aufgrund des kompositionellen Ansatzes beim AHP nicht abbilden. Zwar erlaubt der AHP Aussagen über die Wichtigkeit und Rangordnung der Attribute, jedoch nicht im Sinne der Grenzrate der Substitution, da diese beim AHP nicht vom Niveau der anderen Attribute anhängig gemacht werden kann.

\section{Zusammenfassung: Gremien- und Managemententscheidungen}

Bei der Anwendung des AHP ist zu berücksichtigen, dass die Erfüllung des Transitivitätsaxioms eine notwendige, aber keine hinreichende Voraussetzung ist [40]. Damit werden auch inkonsistente Präferenzurteile bis zu einem gewissen Grad akzeptiert [18]. Die fehlende nutzentheoretische Fundierung und die mangelnde Stabilität der Ergebnisse sind zentrale Kritikpunkte, die bei der Anwendung des AHP zur Präferenzmessung ebenfalls beachtet werden müssen. Die begrenzte Durchführbarkeit von Bewertungen infolge der Skala sowie die Realitätsferne der paarweisen Vergleiche stehen zudem, wie aufgezeigt wurde, im Fokus der Kritik [9, 13, 37]. 


\subsection{Partizipative Entscheidungsfindung}

Positiv könnte sich die Kenntnis der mittels des AHPs erzielten Prioritätsdaten dagegen auf die Arzt-PatientenKommunikation auswirken [31]. Der AHP kann einen Beitrag zur Förderung der partizipativen Entscheidungsfindung leisten, da er einen Rahmen schafft, in dem Ziele definiert, verfügbare Informationen zusammengefasst, Informationsbedarfe priorisiert und letztendlich die Prioritäten hinsichtlich der Entscheidungskriterien gemessen werden. Allein der Prozess der Entscheidungsfindung fördert die sinnvolle Kommunikation zwischen klinischem Entscheidungsträger und dem Patienten bzw. einer Gruppe von Patienten. Der AHP hat das Potential, die Erwartungen und Bedürfnisse der Patienten zu strukturieren. Dolan (1995) kam zu dem Schluss, dass Patienten durchaus in der Lage sind, mit Hilfe des AHP komplexe klinische Probleme zu analysieren [47]. So kann der AHP Unterstützung bei komplexen Entscheidungen und der Implementierung gemeinsamer Arzt-Patienten-Entscheidungen bieten [47]. In diesem Kontext kann die Anwendung des AHP vor allem dann sinnvoll sein, wenn die Erwartungen und Bedürfnisse der Patienten sehr stark variieren oder sich die Prioritäten der Patienten von den Urteilen der Ärzte stark unterscheiden [21]. So schafft der AHP einen praktischen Ansatz, durch den die partizipative Entscheidungsfindung im Gesundheitswesen gefördert und die Verständigung zwischen Leistungserbringern und Leistungsempfängern im Gesundheitswesen verbessert werden kann.

\subsection{Management- und Regulierungsentscheidungen}

Der AHP liefert ein systematisches Verfahren, mit dessen Hilfe Expertenentscheidungen vereinfacht und rationaler getroffen werden können. Ein zentrales und zukünftiges Einsatzgebiet des AHP wird in der Dokumentation von Expertenurteilen von Gesundheitstechnologien und Gesundheitsleistungen im Rahmen von Regulierungsentscheidungen gesehen (wie z. B. Health Technology Assessment HTA) [49]. Vor allem die frühe Nutzenbewertung von Arzneimitteln stellt dabei ein potentielles Anwendungsfeld für den AHP dar [21, 50]. Der HTA-Prozess gliedert sich in verschiedene Phasen, die im Bewertungsprozess durchlaufen werden. So können multikriterielle Verfahren sowohl bei der Priorisierung von Indikationsgebieten oder Projekten in der forschenden Industrie, beim Assessment (dazu zählt die Identifikation und Operationalisierung von patientenrelevanten Endpunkten, der Synthese der multikriteriellen Zielkriterien und der Aggregation multipler Zielgrößen) sowie auch im Rahmen des Appraisals (Entscheidungsfindung) zum Einsatz kommen [49, 51]. Dies aber eher bei der Bewertung durch Expertengruppen, als bei der Dokumentation von Patienten-, Versicherten- oder Bürgerpräferenzen.
Aus der Kritik und den Limitationen dieser Methode resultieren eine Reihe von Anforderungen und Bedingungen an zukünftige Untersuchungen auf diesem Gebiet. Die Methodik ist in Abhängigkeit vom Entscheidungskontext und infolge individueller Erfahrungen, Vorwissen und sonstigen Einflussfaktoren anfällig für systematische Verzerrungen. Um dies zu vermeiden, muss bei der Anwendung stets sichergestellt werden, dass ein geschlossenes Entscheidungsmodell vorliegt. Es müssen alle entscheidungsrelevanten Ziele sowie alle möglichen Alternativen, die der Untersuchungsgegenstand umfasst, Berücksichtigung finden. Da sich die Anwendung des AHP zur Ermittlung von Patientenpräferenzen - nicht zuletzt aufgrund der dargelegten $\mathrm{Li}$ mitationen - schwierig gestaltet, können gewonnene Ergebnisse die realen Präferenzen unter Umständen nur begrenzt widerspiegeln.

Somit besitzt der AHP grundsätzlich die Fähigkeit, die Qualität klinischer Entscheidungen unter Experten zu verbessern und die bestehenden Hemmnisse in der Umsetzung partizipativer Entscheidungsfindungen zu überwinden [31, 47, 48]. Auch könnten die gewonnenen Informationen die klinische und regulatorische Entscheidungsfindung in Expertengruppen sowie auch die Qualität von Entscheidungsprozessen positiv beeinflussen. Einige Autoren kommen so zu dem Schluss, dass der AHP aufgrund der vielfältigen Anwendungen auf medizinische und gesundheitsökonomische Fragestellungen ein erfolgversprechendes Instrument zur multikriteriellen Entscheidungsunterstützung im Gesundheitswesen darstellt [18, 47]. Wesentliche Einsatzgebiete liegen nicht - wie fälschlicherweise gefordert - in der Messung von Patientenpräferenzen, sondern in der Entscheidungsunterstützung von Gremien in der Industrie oder der Selbstverwaltung.

Open Access Dieser Artikel unterliegt den Bedingungen der Creative Commons Attribution License. Dadurch sind die Nutzung, Verteilung und Reproduktion erlaubt, sofern der/die Originalautor/en und die Quelle angegeben sind.

\section{Literatur}

1. Hummel MJM, et al. A multicriteria decision analysis of augmentative treatment of upper limbs in persons with tetraplegia. $\mathrm{J}$ Rehabil Res Dev. 2005;42(5):635-44.

2. Mühlbacher A, Juhnke C. Patient preferences versus physicians' judgements: does it make a difference in health care decisionmaking? Appl Health Econ Health Policy. 2013;11(3):163-80.

3. Mühlbacher AC, et al. Der Wert von Innovationen im Gesundheitswesen: Spielen die Patientenpräferenzen eine Rolle? Recht Politik Gesundh.wes. 2008;14(2):53-62.

4. Glaeske G. The dilemma between efficacy as defined by regulatory bodies and effectiveness in clinical practice. Dtsch Ärztebl. 2012;109(7):115.

5. Mühlbacher AC, Bethge S, Tockhorn A. Präferenzmessung im Gesundheitswesen: Grundlagen von Discrete-Choice Experimenten. Gesundh.ökon Qual.manag. 2013;18(4):159-72. 
6. IQWiG (Institut für Qualität und Wirtschaftlichkeit im Gesundheitswesen). Aktualisierung einiger Abschnitte der Allgemeinen Methoden, Version 4.0, sowie neue Abschnitte zur Erstellung der Allgemeinen Methoden, Version 4.1; 2013.

7. Loh A, et al. Patientenbeteiligung bei medizinischen Entscheidungen. Dtsch Ärztebl. 2007;104(21):1483-8.

8. Saaty TL. The analytic hierarchy process: planning, priority setting, resource allocation. 2. Aufl. New York: McGraw-Hill; 1980. S. XIII, 287.

9. Manthey L. Methoden der Präferenzmessung. Grundlagen, Konzepte und experimentelle Untersuchungen. Norderstedt: Universität Jena; 2007. S. VIII, 212.

10. Saaty TL, Vargas LG. In: Decision making with the analytic network process. Economic, political, social and technological applications with benefits, opportunities, costs and risks. International series in operations research \& management science. Bd. 95. Boston: Springer; 2006.

11. Sarges F. Direkte versus indirekte Präferenzmessung. Ein Vergleich des Analytic Hierarchy Process mit der Conjoint Analyse. Saarbrücken: VDM-Verl. Dr. Müller; 2008. S. IV, 65, XXII.

12. Helm R, Steiner M. Präferenzmessung: Methodengestützte Entwicklung zielgruppenspezifischer Produktinnovationen. Stuttgart: W. Kohlhammer Verlag; 2008.

13. von Nitzsch R. Analytic Hierarchy Process und multiattributive Werttheorie im Vergleich. WiSt Wirtschaftswiss Stud. 1993;22(3):111-6.

14. Ijzerman MJ, van Til JA, Snoek GJ. Comparison of two multicriteria decision techniques for eliciting treatment preferences in people with neurological disorders. Patient - Patient-Centr Outcomes Res. 2008;1(4):265-72.

15. Saaty TL. A scaling method for priorities in hierarchical structures. J Math Psychol. 1977;15(3):234-81.

16. Dolan JG, Isselhardt BJ, Cappuccio JD. The analytic hierarchy process in medical decision making - a tutorial. Med Decis Mak. 1989;9(1):40-50.

17. Dolan JG. Medical decision making using the analytic hierarchy process choice of initial antimicrobial therapy for acute pyelonephritis. Med Decis Mak. 1989;9(1):51-6.

18. Neidhardt K, Wasmuth T, Schmid A. Die Gewichtung multipler patientenrelevanter Endpunkte - Ein methodischer Vergleich von Conjoint Analyse und Analytic Hierarchy Process unter Berücksichtigung des Effizienzgrenzenkonzepts des IQWiG. Universität Bayreuth, Rechts- und Wirtschaftswissenschaftliche Fakultät; 2012.

19. Danner M, et al. Integrating patients' views into health technology assessment: analytic hierarchy process (AHP) as a method to elicit patient preferences. Int J Technol Assess Health Care. 2011;27(04):369-75.

20. Liberatore MJ, Nydick RL. The analytic hierarchy process in medical and health care decision making: a literature review. Eur J Oper Res. 2008;189(1):194-207.

21. Hummel M, Ijzerman M. The past and future of the AHP in health care decision making. In: XI international symposium on the analytic hierarchy process, ISAHP 2011, Sorrento, Naples, Italy; 2011.

22. Saaty TL. Decision making with the analytic hierarchy process. Int J Serv Sci. 2008;1(1):83-98.

23. Saaty TL. How to make a decision: the analytic hierarchy process. Eur J Oper Res. 1990;48(1):9-26.

24. Haedrich G, Kuß A, Kreilkamp E. Der analytic hierarchy process. WiSt Wirtschaftswiss Stud. 1986;3:120-6.

25. Klein R, Scholl A. In: Planung und Entscheidung: Konzepte, Modelle und Methoden einer modernen betriebswirtschaftlichen Entscheidungsanalyse. 2. Aufl. München: Vahlen; 2011.

26. Millet I, Saaty TL. On the relativity of relative measures accommodating both rank preservation and rank reversals in the AHP. Eur J Oper Res. 1998;121:205-12.
27. Chandran B, Golden B, Wasil E. Linear programming models for estimating weights in the analytic hierarchy process. Comput Oper Res. 2005;32(9):2235-54.

28. Harker PT, Vargas LG. The theory of ratio scale estimation: Saaty's analytic hierarchy process. Manag Sci. 1987;33(11): 1383-403.

29. Belton V. A comparison of the analytic hierarchy process and a simple multi-attribute value function. Eur J Oper Res. 1986;26(1):7-21.

30. Helm R, et al. Empirical evaluation of preference elicitation techniques from marketing and decision analysis. Jenaer Schr Wirtsch. 2003.

31. Dolan JG. Shared decision-making - transferring research into practice: the analytic hierarchy process (AHP). Patient Educ Couns. 2008;73(3):418.

32. van Til JA, et al. The use of the analytic hierarchy process to aid decision making in acquired equinovarus deformity. Arch Phys Med Rehabil. 2008;89(3):457-62.

33. Saaty TL. Fundamentals of decision making and prority theory with the analytic hierarchy process. 1. Aufl. Analytic hierarchy process series. Bd. 6. Pittsburgh: RWS Publications; 1994. S. XIV, 527.

34. Saaty TL. Eigenvector and logarithmic least squares. Eur J Oper Res. 1990;48(1):156-60.

35. Tscheulin DK. Analytic hierarchy process. In: Herrmann A, Homburg C, Hrsg. Marktforschung: Methoden, Anwendungen, Praxisbeispiele. Gabler: Wiesbaden; 2000. S. 597-606.

36. Saaty TL. Decision making for leaders: the analytic hierarchy process for decisions in a complex world. 3. Aufl. Pittsburg: RWS Publications; 1995.

37. Dyer JS, Wendell RE. A critique of the analytic hierarchy process. Department of Management, College of Business Administration and Graduate School of Business, University of Texas at Austin; 1985. Working paper 84/85-4-24.

38. Dyer JS. Remarks on the analytic hierarchy process. Manag Sci. 1990;36(3):249-58.

39. Johal SS, Williams HC. Decision-making methods that could be used to assess the value of medical devices; 2005.

40. Scholl A, et al. Solving multiattribute design problems with analytic hierarchy process and conjoint analysis: an empirical comparison. Eur J Oper Res. 2005;164(3):760-77.

41. Brinkmeyer D, Müller RAE. Entscheidungsunterstützung mit dem AHP. Z Agrarinform. 1994;5:82-92.

42. Sommerhäuser G. Unterstützung bankbetrieblicher Entscheidungen mit dem Analytic-Hierarchy-Process. Bd. 170. Berlin: Duncker \& Humblot; 2000.

43. Belton V, Gear T. On a short-coming of Saaty's method of analytic hierarchies. Omega. 1983;11(3):228-30.

44. Watson SR, Freeling ANS. Comment on: Assessing attribute weights by ratios. Omega. 1983;11(1):13.

45. Schoner B, Wedley WC. Ambiguous criteria weights in AHP: consequences and solutions. Decis Sci. 1989;20(3):462-75.

46. Saaty TL, Vargas LG. Models, methods, concepts \& applications of the analytic hierarchy process. International series in operations research \& management science. Boston: Kluwer Academic; 2001. S. 333.

47. Dolan JG. Are patients capable of using the analytic hierarchy process and willing to use it to help make clinical decisions? Med Decis Mak. 1995;15(1):76-80.

48. Dolan JG. Multi-criteria clinical decision support: a primer on the use of multiple criteria decision making methods to promote evidence-based, patient-centered healthcare. Patient. 2010;3(4):229-48.

49. Santos FA, Garcia R. Decision process model to the health technology incorporation. In: Conf proc IEEE eng med biol soc; 2010. S. 414-7. 
50. Hummel M, et al. Applying the AHP in health economic evaluations of new technology. In: XI international symposium on the analytic hierarchy process, ISAHP 2011, Sorrento, Naples, Italy; 2011.

51. Perleth M, Busse R. Health Technology Assessment (HTA) Teil und Methode der Versorgungsforschung. Gesundh.ökon Qual.manag. 2004;9(03):172-6.

52. IQWIG. Analytic Hierarchy Process (AHP) - Pilotprojekt zur Erhebung von Patientenpräferenzen in der Indikation Depression, Version 1.0; 2013.

53. Dintsios CM. Patientengewichtete Endpunkte: Conjoint Analyse (CA) versus Analytic Hierarchy Process (AHP). Monit Versorg.forsch Kongr-Spec. 2013;6(1):16-20.

54. Steiner M. Nachfrageorientierte Präferenzmessung. Wiesbaden: DUV; 2007.

55. Angelucci E, et al. Italian Society of Hematology practice guidelines for the management of iron overload in thalassemia major and related disorders. Haematologica. 2008;93(5):741-52.

56. Balestra G, et al. AHP for the acquisition of biomedical instrumentation. In: Conf proc IEEE eng med biol soc; 2007. S. 3581-4.

57. Barosi $\mathrm{G}$, et al. A unified definition of clinical resistance/ intolerance to hydroxyurea in essential thrombocythemia: results of a consensus process by an international working group. Leukemia. 2007;21(2):277-80.

58. Baykasoglu A, Dereli T, Yilankirkan N. Application of cost/benefit analysis for surgical gown and drape selection: a case study. Am J Infect Control. 2009;37(3):215-26.

59. Benaim $\mathrm{C}$, et al. Using an analytical hierarchy process (AHP) for weighting items of a measurement scale: a pilot study. Rev Epidemiol Sante Publique. 2010;58(1):59-63.

60. $\mathrm{Hu} \mathrm{H}$, et al. Establishment and evaluation of a model of a community health service in an underdeveloped area of China. Public Health. 2010;124(4):206-17.

61. Wang KI, et al. Analysis of senior medical students' preferences in specialty choice - a survey in a medical school in northern Taiwan. Chang Gung Med J. 2007;30(4):339-53.

62. Cheever MA, et al. The prioritization of cancer antigens: a national cancer institute pilot project for the acceleration of translational research. Clin Cancer Res. 2009;15(17):5323-37.

63. Cho KT, Kim SM. Selecting medical devices and materials for development in Korea: the analytic hierarchy process approach. Int J Health Plann Manage. 2003;18(2):161-74.

64. Chu HL, Wang CC, Dai YT. A study of a nursing department performance measurement system: using the balanced scorecard and the analytic hierarchy process. Nurs Econ. 2009;27(6):401-7.

65. Chung KP, et al. Application of the analytic hierarchy process in the performance measurement of colorectal cancer care for the design of a pay-for-performance program in Taiwan. Int J Qual Health Care. 2013;25(1):81-91.

66. Czaja SJ, et al. A methodology for describing and decomposing complex psychosocial and behavioral interventions. Psychol Aging. 2003;18(3):385.

67. Dulin MF, et al. Using geographic information systems (GIS) to understand a community's primary care needs. J Am Board Fam Med. 2010;23(1):13-21.

68. Fang LF, Tung HH. Comparison of nurse practitioner job core competency expectations of nurse managers, nurse practitioners, and physicians in Taiwan. J Am Acad Nurse Pract. 2010;22(8):409-16.

69. Gomi Y, Nogawa H, Tanaka H. Toward secure distribution of electronic health records: quantitative feasibility study on secure e-mail systems for sharing patient records. J Med Dent Sci. 2005;52(4):229-36.

70. Gurtner S. Making the right decisions about new technologies: a perspective on criteria and preferences in hospitals. Health Care Manage Rev. 2013.
71. Hariharan S, et al. A new tool for measurement of process-based performance of multispecialty tertiary care hospitals. Int J Health Care Qual Assur Inc Leadersh Health Serv. 2004;17(6):302-12.

72. Hariharan S, et al. Application of analytic hierarchy process for measuring and comparing the global performance of intensive care units. J Crit Care. 2005;20(2):117-24.

73. Hilgerink MP, et al. Assessment of the added value of the twente photoacoustic mammoscope in breast cancer diagnosis. Med Devices. 2011;4:107-15.

74. Ho CC. Optimal evaluation of infectious medical waste disposal companies using the fuzzy analytic hierarchy process. Waste Manag. 2011;31(7):1553-9.

75. Hsu PF, Wu CR, Li YT. Selection of infectious medical waste disposal firms by using the analytic hierarchy process and sensitivity analysis. Waste Manag. 2008;28(8):1386-94.

76. Hsu HC, et al. Constructing area-level indicators of successful ageing in Taiwan. Health Soc Care Community. 2010;18(1):7081.

77. Hummel JM, et al. Predicting the health economic performance of new non-fusion surgery in adolescent idiopathic scoliosis. J Orthop Res. 2012;30(9):1453-8.

78. Joshi V, et al. Empirical investigation of radiologists' priorities for PACS selection: an analytical hierarchy process approach. J Digit Imaging. 2011;24(4):700-8.

79. Kim K, et al. Efficient management design for swimming exercise treatment. Korean J Physiol Pharmacol. 2009;13(6):497502.

80. Krishnan JA, et al. Stakeholder priorities for comparative effectiveness research in chronic obstructive pulmonary disease: a workshop report. Am J Respir Crit Care Med. 2013;187(3):320-6.

81. Lee BM, Park RW, Choi JT. The order of priority in standardization of ubiquitous home healthcare technology. In: AMIA annu symp proc; 2007. S. 1024.

82. Lin RH, Chuang CL. A hybrid diagnosis model for determining the types of the liver disease. Comput Biol Med. 2010;40(7):665-70.

83. Low C, Chen YH. Criteria for the evaluation of a cloud-based hospital information system outsourcing provider. J Med Syst. 2012;36(6):3543-53.

84. Maino M, et al. The analytic hierarchy process in decisionmaking for caprine health programmes. Rev Sci Technol. 2012;31(3):889-97.

85. Olivieri A, et al. Proposed definition of 'poor mobilizer' in lymphoma and multiple myeloma: an analytic hierarchy process by ad hoc working group Gruppo Italiano Trapianto di Midollo Osseo. Bone Marrow Transplant. 2012;47(3):342-51.

86. Page K. The four principles: can they be measured and do they predict ethical decision making? BMC Med Ethics. 2012;13:10.

87. Pecchia L, et al. Analytic hierarchy process (AHP) for examining healthcare professionals' assessments of risk factors: the relative importance of risk factors for falls in community-dwelling older people. Methods Inf Med. 2011;50(5):435-44.

88. Pecchia $\mathrm{L}$, et al. User needs elicitation via analytic hierarchy process (AHP): a case study on a computed tomography (CT) scanner. BMC Med Inform Decis Mak. 2013;13:2.

89. Shin T, et al. The comparative evaluation of expanded national immunization policies in Korea using an analytic hierarchy process. Vaccine. 2009;27(5):792-802.

90. Smith J, Cook A, Packer C. Evaluation criteria to assess the value of identification sources for horizon scanning. Int J Technol Assess Health Care. 2010;26(3):348-53.

91. Suner A, et al. Sequential decision tree using the analytic hierarchy process for decision support in rectal cancer. Artif Intell Med. 2012;56(1):59-68. 
92. Sylvie AK, Jiang Q, Cohen N. Identification of environmental supports for healthy eating in older adults. J Nutr Gerontol Geriatr. 2013;32(2):161-74.

93. Sushil, TU. E-healthcare in India: critical success factors for sustainable health systems. Stud Health Technol Inform. 2007;129(Pt 1):257-61.

94. Ajami S, Ketabi S. Performance evaluation of medical records departments by analytical hierarchy process (AHP) approach in the selected hospitals in Isfahan. J Med Syst. 2012;36(3):116571.

95. Chang P-Y, et al. Factors influencing medical students' choice of specialty. J Formos Med Assoc. 2006;105(6):489-96.

96. Ricci A, et al. Raw milk-associated foodborne infections: a scoring system for the risk-based categorisation of raw dairy farms. Res Vet Sci. 2013;95(1):69-75.

97. Shibata T, Shimizu Y, Wada H. Psychological evaluation of 24hour home nursing care: application of an analytic hierarchy process to improve quality of life. Stud Health Technol Inform. 2012;180:1138-40.

98. Nuijten MJ, Kosa J. Pricing of pharmaceuticals: assessing the pricing potential by a pricing matrix model. Eur J Health Econ. 2004;5(2):110-5.

99. Katsumura Y, et al. Relationship between risk information on total colonoscopy and patient preferences for colorectal cancer screening options: analysis using the analytic hierarchy process. BMC Health Serv Res. 2008;8:106.

100. Mochimaru M, et al. Questionnaire survey of customer satisfaction for product categories towards certification of ergonomic quality in design. Work. 2012;41(Suppl 1):956-9.

101. Tan X, et al. Evaluation of the effect of a health education campaign of HIV by using an analytical hierarchy process method. Int J Environ Res Public Health. 2007;4(3):254-9.
102. Basoglu N, Daim TU, Topacan U. Determining patient preferences for remote monitoring. J Med Syst. 2012;36(3):1389401.

103. Dolan JG, et al. Patients' preferences and priorities regarding colorectal cancer screening. Med Decis Mak. 2013;33(1):59-70.

104. Dolan JG, Iadarola S. Risk communication formats for low probability events: an exploratory study of patient preferences. BMC Med Inform Decis Mak. 2008;8:14.

105. Hummel MJ, et al. Using the analytic hierarchy process to elicit patient preferences: prioritizing multiple outcome measures of antidepressant drug treatment. Patient. 2012;5(4):225-37.

106. Ijzerman MJ, van Til JA, Bridges JF. A comparison of analytic hierarchy process and conjoint analysis methods in assessing treatment alternatives for stroke rehabilitation. Patient. 2012;5(1):45-56.

107. Kitamura Y. Decision-making process of patients with gynecological cancer regarding their cancer treatment choices using the analytic hierarchy process. Jpn J Nurs Sci. 2010;7(2):148-57.

108. Richman MB, et al. A novel computer based expert decision making model for prostate cancer disease management. J Urol. 2005;174(6):2310-8.

109. Sharma PS, et al. Subjective risk vs. objective risk can lead to different post-cesarean birth decisions based on multiattribute modeling. J Clin Epidemiol. 2011;64(1):67-78.

110. Tzung TY, et al. Decision factors and the recognition of medical specialty in patients receiving cosmetic laser and intense pulsed light treatment. Dermatol Surg. 2007;33(12):1488-93.

111. Uzoka FM, et al. An experimental comparison of fuzzy logic and analytic hierarchy process for medical decision support systems. Comput Methods Programs Biomed. 2011;103(1):10-27.

112. Wollmann D, et al. Evaluation of health service providers by consumers through the analytic hierarchy process method. Rev Saude Publica. 2012;46(5):777-83. 